\title{
RADIOCHIMICA ACTA
}

SC H R I FT LE ITU NG : A.H.W. Aten jr., Amsterdam • H. J. Born, München

E D ITO R S : E. Glueckauf, Harwell · P. Regnaut, Fontenay-aux-Roses

É D ITÉ PAR: F.Strassmann, Mainz $\cdot$ R. Wolfgang, New Haven

HERAUSGEGEBEN VON:

EDITORIAL BOARD: COMITÉ DE RÉDACTION:
F. Baumgäriner, Heidelberg - G. E. Boyd, Oak Ridge

Ch. Fisher, Saclay - J. Flegenheimer, Buenos Aires

A. Friedman, Argonne - H. Götte, Frankfurt a. M.

G. Harbottle, Upton · F. Hecht, Wien - F. Hernegger, Wien

W. Herr, Köln · G. Herrmann, Mainz - E. K. Hyde, Berkeley

K. Kimura, Tokyo - R. Lindner, Braunschweig

W. W. Meinke, Ann Arbor - E. Merz, Jülich - H. Münzel, Karlsruhe A. C. Pappas, Oslo • M. V. Ramaniah, Bombay · N. Riehl, München W.Seelmann-Eggebert, Karlsruhe - A. A. Smales, Harwell

K. Starke, Marburg • J. I. Vargas, Belo Horizonte

M. Zifferero, Rom

B A N D

VOLUME VOLUME

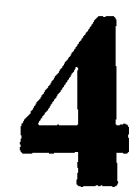

1965 
(C) Akademische Verlagsgesellschaft 1965, - Printed in Germany. - Alle Rechte, insbesondere die der Ubersetzung, vorbehalten. Kein Teil dieser Zeitschrift darf in irgendeiner Form - durch Photokopie. Mikroflm oder irgendein anderes Verfahren - ohne schriftliche Genehmigung des Verlages reproduziert werden. All rights reserved (including those of translations into foreign languages). No part of this issue may be reproduced in any form, by photoprint. microflm or any other means without written permission from the publishers. Herstellung: Universitätsdruckerei Mainz GmbH 